\title{
A Fluorogenic Assay for the Rapid Detection of Some Vibrio Species Including Vibrio parahaemolyticus in Foods
}

(Received June 27, 1989)

\author{
Takahisa Miyamoto*1, Yea-Ing Sheu*1, Harufumi Mrwa ${ }^{* 2}$ \\ and Shoji HATANO*1 \\ $\left({ }^{* 1}\right.$ Department of Food Science and Technology, Faculty of Agriculture, Kyushu University: \\ 6-10-1, Hakozaki, Higashi-ku, Fukuoka, Japan; *2 Ajinomoto Co., Inc.: \\ $1-5-8$, Kyobashi, Chuo-ku, Tokyo, Japan)
}

\begin{abstract}
A rapid assay method for some Vibrio species including Vibrio parahaemolyticus was developed. The assay involved the enrichment culture of Vibrio species in BSP-HP medium ( $0.2 \%$ bacto-tryptone, $2 \%$ sodium chloride, 250 units $/ \mathrm{ml}$ polymy xin B sulfate, $0.025 \%$ sodium hexametaphosphate, $\mathrm{pH} 8.5$ ) and the specific measurement of intracellular trypsin-like activity by using the fluorogenic substrate benzoyl-L-arginine-7-aminomethylcoumarin. In BSP-HP medium, growth and trypsin-like activity of bacteria other than $V$. parahaemolyticus, $V$. alginolyticus and $V$. harveyi were suppressed. Although trypsin-like enzyme in food samples interfered with the fluorogenic assay, the interference could be overcome by the addition of $1 \mathrm{mM}$ EDTA to the reaction mixture. Using this fluorogenic assay with commercial seafoods, 400 cells of $V$. parahaemolyticus per gram of food sample were detected within $8 \mathrm{hr}$. Trypsinlike activity measured by the assay was proportional to the number of $V$. parahaemolyticus determined by the conventional BTB teepol agar plating method (correlation coefficient $r=$ 0.97 ). In the medium, $V$. alginolyticus, one of the $V$. parahaemolyticus-allied bacteria, and $V$. harveyi also showed vigorous growth and high trypsin-like activity. A more specific culture condition for $V$. parahaemolyticus is required, and further investigation is in progress.
\end{abstract}

Key words: Vibrio parahaemolyticus; fluorogenic assay; trypsin-like activity; rapid detection; benzoyl-L-arginine-7-aminomethylcoumarin

\section{Introduction}

Food poisoning in Japan is most frequently due to Vibrio parahaemolyticus. Most of the $V$. parahaemolyticus food poisoning is caused by raw fish and shellfish ${ }^{1)}$. The most common method to detect $V$. parahaemolyticus in marine specimens is a culture procedure which depends on selective media and sometimes requires enrichment for isolation from sparsely-infected materials ${ }^{2}$. With this conventional method, $V$. parahaemolyticus detection requires from two to three days; therefore, there is a requirement for a rapid detection method for use in food quality control.

In recent years, a number of new methods have been developed to increase the efficiency and speed of food microorganism detection while reducing the labor ${ }^{3)}$. The fluorogenic assay, using a fluorogenic substrate for the highsensitivity detection of an enhanced enzymatic activity in bacteria, is one of these methods ${ }^{4}$.

The present study was undertaken to develop rapid, specific, and more efficient detection assays for $V$. parahaemolyticus by using the fluorogenic substrate, benzoyl-L-arginine-7aminomethylcoumarin, to detect intracellular trypsin-like activity.

\section{Materials and Methods}

\section{Strains}

Escherichia coli IFO 3301, Salmonella typhimurium IFO 12529, S. enteritidis IFO 3313, Enterobacter aerogenes IFO 13534, Pseudomonas fragi IFO 3458, Staphylococcus aureus IFO 3060 and Bacillus brevis IFO 3331 were obtained from the Institute for Fermentation, Osaka. Campylobacter jejuni JCM 2013, Bacillus subtilis JCM 
1465, B. circulans JCM 2504, B. cereus JCM 2152, B. pumilus JCM 2508, Lactobacillus lactis JCM 1248 and $L$. heterohiochii JCM 1198 were obtained from the Japan Collection of Microorganisms. Vibrio parahaemolyticus WP-1, V. parahaemolyticus 46-11, V. parahaemolyticus 39-11, V. parahaemolyticus 39-3, V. parahaemolyticus 26-1, V. parahaemolyticus 27-2, V. parahaemolyticus 33-7, V. parahaemolyticus 33-8, V. parahaemolyticus 33-10, $V$. alginolyticus 10-1, $V$. alginolyticus 13-1, V. alginolyticus 14-1, V. cholerae FK, $V$. damsela FK, $V$. vulnificus $\mathrm{FK}$ and $V$. harveyi $\mathrm{FK}$ were obtained from Fukuoka City Institute of Public Health. V. cholerae non 0-1 NR, V. fuvialis NR and $V$. furnissii NR were provided by Dr. Seiichi Umesako, Nara Prefectural Institute of Public Health. V. anguillarum Ty 12, Alteromonas communis IAM 12914, A. undina IAM 12922 and Aeromonas hydrophila A 0111003 were kindly provided by Prof. Ushio Shimizu, Ocean Research Institute, University of Tokyo.

\section{Fluorogenic substrate}

The 4 -methylumbelliferone (4-MU) derivatives shown in Table 1 were purchased from either Nacalai Tesque, Inc., or Polyscience, Inc. The 7-aminomethylcoumarin (7AMC) derivatives were obtained from Peptide Institute, Inc., Osaka. These fluorogenic substrates were dissolved at a concentration of $6 \mathrm{mM}$ in dimethyl sulfoxide and kept at a temperature of $-20^{\circ} \mathrm{C}$.

\section{Media}

All of the media were sterilized in an autoclave. These media included PM broth (1\% polypeptone, $1 \%$ meat extract, $\mathrm{pH} 7.0)$ and BSP-HP medium $(0.2 \%$ bacto-tryptone, $2 \%$ sodium chloride, 250 units $/ \mathrm{ml}$ polymyxin B sulfate, $0.025 \%$ sodium hexametaphosphate, $\mathrm{pH}$ 8.5).

\section{Selection and measurement of a marker enzyme for $V$. parahaemolyticus detection}

Various bacteria from slant cultures for preservation were inoculated in PM broth and cultured overnight at $30^{\circ} \mathrm{C}$. This broth with $2 \%$ sodium chloride added was used to cultivate $V$. parahaemolyticus. To measure enzyme activity, the culture broth was diluted to $A_{660}=0.02$ with PM broth. When there was no bacterial growth, undiluted culture broth was used to measure enzyme activity. Bacterial cells in $7 \mathrm{ml}$ of the culture broth were collected by centrifugation $(1,400 \times \mathrm{g}$ for $10 \mathrm{~min}) ; 3 \mathrm{ml}$ of $50 \mathrm{mM}$ phosphate buffer ( $\mathrm{pH} 7.5$ ) including $0.01 \mathrm{mM}$ fluorogenic substrate was added to the precipitated cell mass; $20 \mu \mathrm{l}$ of toluene was added for lysis; and the reaction was allowed to proceed for $1 \mathrm{hr}$ at $40^{\circ} \mathrm{C}$. The reaction was stopped by addition of 1 $\mathrm{ml}$ of $1 M$ glycine buffer ( $\mathrm{pH} 11.0)$ and after centrifugation $(1,400 \times \mathrm{g}$ for $10 \mathrm{~min})$, the fluorescence intensity of the supernatant was measured with a Shimadzu RF-520 fluorospectrometer. The excitation wavelength was $360 \mathrm{~nm}$ and fluorescence intensity was measured at 450 nm. A control, including all components of the reaction mixture except the cultured bacterial cells, was assayed with each sample. The enzyme activity was expressed as the fluorescence intensity of the test sample relative to that of the broth control, which was set at 50 .

\section{Preparation of food samples}

Five grams of each food sample was homog-

Table 1. List of Fluorogenic Substrates

\begin{tabular}{ll}
\hline \hline Compound & Degradation enzyme \\
\hline 4-MU- $\alpha$-L- & Arabinosidase \\
arabinopyranoside & \\
4-MU- $\beta$-D-galactoside & Galactosidase \\
4-MU- $\alpha$-D-glucoside & $\alpha$-Glucosidase \\
4-MU- $\beta$-D-glucoside & $\beta$-Glucosidase \\
4-MU- $\beta$-D-glucuronide & Glucuronidase \\
4-MU- $\alpha$-D- & Mannosidase \\
mannopyranoside & \\
4-MU- $\beta$-D- & Xylosidase \\
xylopyranoside & \\
4-MU-phosphate, & Phosphatase \\
disodium salt & \\
4-MU-sulfate, & Sulfatase \\
potassium salt & \\
Bz-Arg-7AMC & Trypsin-like enzyme \\
Leu-7AMC & Arylaminopeptidase \\
Pyr-7AMC & Pyroglutamyl peptidase \\
Suc-Ala-Pro-Ala- & Elastase \\
7AMC & \\
Suc-Gly-Pro-7AMC & Post-proline cleaving enzyme \\
Suc-Leu-Leu-Val-Tyr- & Chymotrypsin-like enzyme \\
7AMC & \\
\hline 4-MU: $4-$ AMethylumbeliferone; & \\
\hline
\end{tabular}
4-MU: 4-methylumbelliferone; 7AMC: 7-aminomethylcoumarin 
Table 2. Enzyme Activities of Various Bacteria

\begin{tabular}{|c|c|c|c|c|c|c|c|c|c|c|c|c|c|c|c|}
\hline \multirow{2}{*}{ Strain } & \multicolumn{15}{|c|}{ Activity } \\
\hline & Ar & Ga & $\alpha \mathrm{G}$ & $\beta G$ & Gl & $\mathrm{Ma}$ & $\mathrm{Xy}$ & $\mathrm{Am}$ & $\mathrm{Ch}$ & $\mathrm{El}$ & Py & Po & $\operatorname{Tr}$ & $\mathrm{Ph}$ & $\mathrm{Su}$ \\
\hline $\begin{array}{l}\text { Control } \\
\text { gram-negative }\end{array}$ & 1.0 & 1.0 & 1.0 & 1.0 & 1.0 & 1.0 & 1.0 & 1.0 & 1.0 & 1.0 & 1.0 & 1.0 & 1.0 & 1.0 & 1.0 \\
\hline V. parahaemolyticus WP-1* & 1.3 & 1.0 & 1.2 & 1.1 & 0.9 & 1.6 & 1.3 & 17.6 & 1.3 & 1.5 & 0.8 & 2.5 & 55.5 & 0.8 & 1.1 \\
\hline C. jejuni JCM 2013 & 1.3 & 63.2 & 12.0 & 5.2 & 1.0 & 4.1 & 1.2 & 5.5 & 3.3 & 1.4 & 1.5 & 1.2 & 1.7 & 1.0 & 1.0 \\
\hline E. aerogenes IFO 13534 & 3.8 & 66.0 & 1.7 & 4.1 & 1.0 & 1.2 & 3.6 & 6.3 & 1.8 & 1.5 & 1.7 & 1.1 & 2.0 & 6.3 & 1.0 \\
\hline E. coli IFO 3301 & 10.31 & 109.0 & 1.3 & 1.3 & 104.6 & 1.1 & 1.0 & 11.4 & 1.9 & 1.3 & 1.0 & 1.1 & 7.5 & 7.6 & 1.0 \\
\hline P. fragi IFO 3458 & 1.3 & 1.2 & 1.1 & 1.0 & 1.1 & 1.2 & 0.9 & 2.8 & 7.2 & 0.9 & 2.1 & 1.1 & 1.8 & 0.8 & 0.8 \\
\hline S. enteritidis IFO 3313 & 1.0 & 1.1 & 1.7 & 1.4 & 0.8 & 0.9 & 1.7 & 7.4 & 1.1 & 1.5 & 1.1 & 1.2 & 3.3 & 3.9 & 1.2 \\
\hline $\begin{array}{l}\text { S. typhimurium IFO } 12529 \\
\text { gram-positive }\end{array}$ & 1.3 & 0.9 & 1.1 & 1.2 & 0.8 & 0.8 & 1.0 & 7.3 & 0.9 & 1.0 & 1.0 & 1.2 & 3.2 & 7.2 & 0.9 \\
\hline B. brevis IFO 3331 & 1.0 & 1.2 & 1.1 & 1.0 & 1.2 & 1.0 & 1.7 & 2.1 & 2.5 & 1.4 & 3.1 & 1.6 & 1.9 & 1.1 & 1.1 \\
\hline B. cereus JCM 2152 & 1.1 & 1.3 & 26.0 & 1.1 & 1.1 & 1.2 & 1.3 & 3.3 & 2.1 & 1.0 & 1.3 & 1.4 & 1.3 & 7.5 & 1.0 \\
\hline B. circulans JCM 2504 & 38.3 & 30.8 & 60.6 & 18.8 & 1.1 & 16.81 & 75.0 & 2.3 & 1.4 & 1.2 & 1.5 & 1.2 & 1.3 & 1.0 & 1.0 \\
\hline B. pumilus JCM 2508 & 1.0 & 7.0 & 0.8 & 64.0 & 1.1 & 1.0 & 2.8 & 2.3 & 3.6 & 1.0 & 1.4 & 1.1 & 1.2 & 1.0 & 1.1 \\
\hline B. subtilis JCM 1465 & 1.3 & 3.7 & 123.9 & 28.9 & 1.0 & 1.4 & 28.0 & 2.22 & 209.8 & 1.4 & 2.7 & 1.2 & 1.3 & 1.0 & 1.0 \\
\hline L. heterohiochii JCM 1198 & 1.4 & 1.6 & 34.0 & 1.2 & 1.0 & 1.1 & 1.2 & 9.2 & 2.3 & 1.6 & 2.8 & 1.5 & 1.0 & 1.8 & 1.0 \\
\hline L. lactis JCM 1248 & 0.9 & 1.3 & 21.2 & 1.2 & 1.1 & 1.5 & 1.01 & 11.3 & 3.1 & 1.4 & 3.1 & 1.3 & 1.6 & 1.4 & 0.9 \\
\hline S. aureus IFO 3060 & 1.0 & 1.1 & 3.0 & 1.1 & 0.9 & 1.4 & 0.9 & 4.0 & 1.5 & 1.4 & 1.8 & 1.1 & 1.7 & 14.5 & 0.8 \\
\hline
\end{tabular}

Medium: $1 \%$ polypeptone, $1 \%$ meat extract, $\mathrm{pH} 7.0$.

* Medium: $1 \%$ polypeptone, $1 \%$ meat extract, $2 \% \mathrm{NaCl}, \mathrm{pH} 7.0$.

Abbreviations: Ar, arabinosidase; Ga, galactosidase; $\alpha \mathrm{G}, \alpha$-glucosidase; $\beta \mathrm{G}, \beta$-glucosidase; Gl, glucuronidase; Ma, mannosidase; Xy, xylosidase; Am, arylaminopeptidase; Ch, chymotrypsin-like enzyme; $\mathrm{El}$, elastase; Py, pyroglutamyl peptidase; Po, post-proline cleaving enzyme; $\mathrm{Tr}$, trypsin-like enzyme; $\mathrm{Ph}$, phosphatase; Su, sulfatase

enized with $45 \mathrm{ml}$ of peptone water (1\% polypeptone, $2 \%$ sodium chloride, $\mathrm{pH} 7.0$ ) in a sterilized Waring blender (14,700 rpm for $2 \mathrm{~min})$ and used as an undiluted sample solution ${ }^{2)}$.

\section{Vibrio colony count}

The sample solution was serially diluted with peptone water, and $0.1 \mathrm{ml}$ of the diluted solution was spread evenly over BTB teepol agar (Nissui Pharmaceutical Co., Ltd.). After incubation at $35^{\circ} \mathrm{C}$ for $18 \mathrm{hr}$, typical colonies of $V$. parahaemolyticus and $V$. alginolyticus were counted ${ }^{2}$.

\section{Results}

Determination of a marker enzyme to detect $V$. parahaemolyticus

Fifteen strains of food-poisoning bacteria and food-spoilage bacteria, including $V$. parahaemolyticus, were selected and cultured in PM broth. Each strain was incubated with the substrate shown in Table 1, and 15 different enzyme activities were measured using the fluorogenic assay. The results are shown in Table 2 .
Arylaminopeptidase and trypsin-like activities were highest in $V$. parahaemolyticus. Arylaminopeptidase activity was present in all the bacteria tested, but trypsin-like activity was not observed in gram-positive bacteria. The results suggest that when $V$. parahaemolyticus is cultured in a selective-enrichment medium, arylaminopeptidase or trypsin-like activity can be used as a highly sensitive indicator, by using the fluorogenic assay.

Selective-enrichment medium to detect arylaminopeptidase and trypsin-like activities of $V$. parahaemolyticus

To obtain higher activities of intracellular arylaminopeptidase and trypsin-like enzyme in $V$. parahaemolyticus, enrichment media of 32 different compositions were tested, and BS medium $(0.2 \%$ bacto-tryptone, $2 \%$ sodium chloride, $\mathrm{pH} 8.5$ ) was found to increase the activity of the enzymes. Polymyxin B sulfate (250 units $/ \mathrm{ml}$ ) and $0.025 \%$ sodium hexametaphosphate $(\mathrm{HP})^{5)}$ were added to the BS medium to suppress 
Table 3. Arylaminopeptidase and Trypsin-Like Activities of Various Bacteria Grown in BSP-HP Medium

\begin{tabular}{|c|c|c|c|}
\hline \multirow{2}{*}{$\begin{array}{c}\text { Strain } \\
\text { (Kanagawa hemolysin, Serotype) }\end{array}$} & \multirow{2}{*}{$\begin{array}{l}\text { Growth } \\
\left(A_{660}\right)\end{array}$} & \multicolumn{2}{|c|}{ Activity } \\
\hline & & Arylamino. & Trypsin-like \\
\hline Control & 0 & 1.0 & 1.0 \\
\hline$V$. parahaemolyticus WP-1 (+) & 0.08 & 101.0 & 175.0 \\
\hline V. parahaemolyticus $27-2 \quad(+, \mathrm{K} 7)$ & 0.07 & 109.0 & 166.0 \\
\hline V. parahaemolyticus $33-7 \quad(+, \mathrm{K} 64)$ & 0.07 & 46.0 & 190.0 \\
\hline$V$. parahaemolyticus $33-8 \quad(+, \mathrm{K} 8)$ & 0.09 & 38.0 & 63.0 \\
\hline$V$. parahaemolyticus $33-10(+, \mathrm{K} 38)$ & 0.10 & 75.0 & 121.0 \\
\hline$V$. parahaemolyticus $26-1 \quad(-, \mathrm{K} 41)$ & 0.10 & 81.0 & 148.0 \\
\hline V. parahaemolyticus $39-3 \quad(-, \mathrm{K} 57)$ & 0.10 & 62.0 & 82.0 \\
\hline V. parahaemolyticus 39-11 (-, K13) & 0.08 & 97.0 & 153.0 \\
\hline V. parahaemolyticus 46-11 (-, K8) & 0.10 & 58.0 & 110.0 \\
\hline$V$. alginolyticus $10-1$ & 0.11 & 39.0 & 112.0 \\
\hline V. alginolyticus $13-1$ & 0.11 & 29.0 & 107.0 \\
\hline$V$. alginolyticus $14-1$ & 0.12 & 53.0 & 103.0 \\
\hline V. anguillarum Ту 12 & 0 & 54.5 & 1.0 \\
\hline$V$. cholerae FK & 0 & 29.3 & 1.6 \\
\hline$V$. cholerae non $0-1 \mathrm{NR}$ & 0.01 & 16.8 & 0.9 \\
\hline V. damsela FK & 0 & 13.1 & 1.0 \\
\hline V. fluvialis NR & 0.02 & 130.0 & 1.2 \\
\hline V. furnissii NR & 0 & 36.3 & 2.0 \\
\hline$V$. harveyi $\mathrm{FK}$ & 0.02 & 60.0 & 156.0 \\
\hline V. vulnificus $\mathrm{FK}$ & 0.04 & 36.6 & 1.0 \\
\hline A. hydrophila A 0111003 & 0 & 1.5 & 2.3 \\
\hline A. communis IAM 12914 & 0 & 2.0 & 1.3 \\
\hline A. undina IAM 12922 & 0 & 1.3 & 4.1 \\
\hline C. jejuni JCM 2013 & 0 & 1.3 & 1.1 \\
\hline E. aerogenes IFO 13534 & 0.04 & 41.6 & 8.5 \\
\hline E. coli IFO 3301 & 0 & 18.8 & 4.4 \\
\hline P. fragi IFO 3458 & 0 & 1.9 & 1.5 \\
\hline S. typhimurium IFO 12529 & 0 & 9.8 & 2.7 \\
\hline S. enteritidis IFO 3313 & 0 & 10.7 & 2.5 \\
\hline B. subtilis JCM 1465 & 0 & 1.1 & 1.0 \\
\hline B. circulans JCM 2504 & 0 & 1.2 & 1.6 \\
\hline B. cereus JCM 2152 & 0 & 1.0 & 1.1 \\
\hline B. brevis IFO 3331 & 0 & 1.2 & 1.2 \\
\hline B. pumilus JCM 2508 & 0 & 1.7 & 1.1 \\
\hline L. lactis JCM 1248 & 0 & 1.0 & 1.9 \\
\hline S. aureus IFO 3060 & 0 & 1.0 & 1.0 \\
\hline
\end{tabular}

Various bacteria were inoculated in BSP-HP medium and cultured at $30^{\circ} \mathrm{C}$ for $16 \mathrm{hr}$. Arylaminopeptidase (Arylamino.) and trypsin-like activities were measured by the fluorogenic assay.

the growth of gram-negative bacteria other than $V$. parahaemolyticus and gram-positive bacteria, respectively (BSP-HP medium). In BSP-HP medium, the growth and the enzyme activities of various bacteria were measured, and the results are shown in Table 3. V. parahaemolyticus, regardless of its Kanagawa response or antigen type, exhibited high arylaminopeptidase and trypsin-like activities. $V$. alginolyticus and $V$. harveyi also grew in BSP-HP medium and showed high enzyme activities. $V$. cholerae, $V$. cholerae non 0-1, V. fluvialis, V. damsela, V. vulnificus, $V$. furnissii, $V$. anguillarum, Alteromonas undina, Escherichia coli and Enterobacter aerogenes showed low trypsin-like activity though they exhibited high arylaminopeptidase activity. These results indicate that after being cultured in BSP-HP medium, species of Vibrio such 
as $V$. parahaemolyticus, $V$. alginolyticus and $V$. harveyi can be specifically detected by measuring intracellular trypsin-like activity.

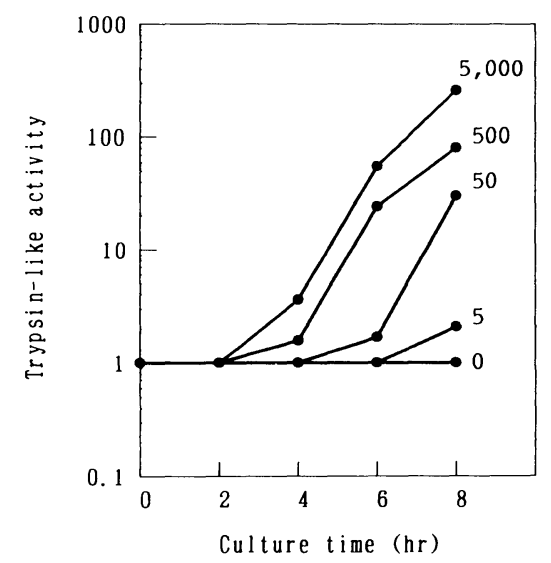

Fig. 1. Time course of trypsin-like activity production

$V$. parahaemolyticus cells were cultured at $30^{\circ} \mathrm{C}$ in a test tube containing $7 \mathrm{ml}$ of BSPHP medium. The culture was centrifuged at $1,400 \times \mathrm{g}$ for $10 \mathrm{~min}$ after a suitable interval. Trypsin-like activity of the precipitate was measured by the fluorogenic assay. Cell number inoculated into the medium is indicated to the right of each curve.
Incubation period required for $V$. parahaemolyticus detection

To determine the minimum incubation period for the detection of trypsin-like activity, 5 to $5 \times$ $10^{3}$ cells of $V$. parahaemolyticus were cultured in $7 \mathrm{ml}$ of BSP-HP medium, and the enzyme activity was measured periodically. The changes in trypsin-like activity are shown in Fig. 1. With the inoculation of $5 \times 10^{2}$ or $5 \times 10^{3}$ cells of $V$. parahaemolyticus, the enzyme activity was detected after incubation for $4 \mathrm{hr}$, and with initial cell numbers of 5 and 50 , the activity was detectable after incubation for 8 and $6 \mathrm{hr}$, respectively.

Trypsin-like activity of $V$. parahaemolyticus, $V$. alginolyticus and $V$. harveyi cultured in BSP-HP medium

Trypsin-like activities of $V$. parahaemolyticus, $V$. alginolyticus and $V$. harveyi were compared when various initial cell numbers $\left(0\right.$ to $\left.10^{7}\right)$ were inoculated in BSP-HP medium. Figure 2 shows trypsin-like activity of $V$. parahaemolyticus, $V$. alginolyticus and $V$. harveyi cultured in BSP-HP medium. In all the strains of $V$. parahaemolyticus and $V$. harveyi, the activity was proportional to

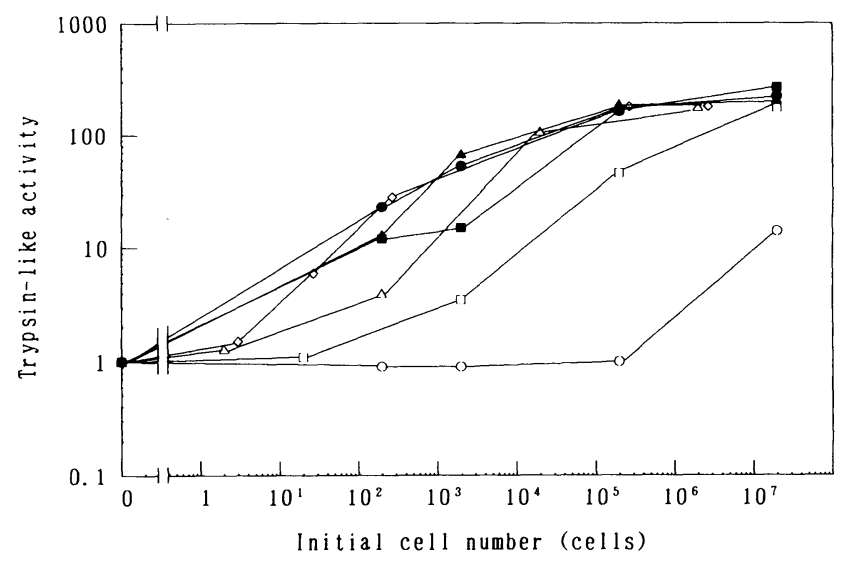

Fig. 2. Trypsin-like activity of $V$. parahaemolyticus, $V$. alginolyticus and $V$. harveyi grown in BSP-HP medium

$V$. parahaemolyticus, $V$. alginolyticus and $V$. harveyi were inoculated in BSP-HP medium $(7 \mathrm{ml})$ at various cell numbers and cultured at $30^{\circ} \mathrm{C}$ for $6 \mathrm{hr}$. The culture broth was centrifuged at $1,400 \times \mathrm{g}$ for $10 \mathrm{~min}$. Trypsin-like activity of the precipitate was measured by the fluorogenic assay.

Symbols: O, V. parahaemolyticus WP-1; $\mathbf{\Delta}, V$. parahaemolyticus $33-10$;

a, V. parahaemolyticus 26-1; $\bigcirc, V$. alginolyticus 10-1;

$\triangle$, V. alginolyticus 13-1; $\square, V$. alginolyticus 14-1;

$\diamond, V$. harveyi $\mathrm{FK}$ 
the initial cell number. In $V$. alginolyticus, however, the activity of the three strains was variable, and in two of the strains it was as high as that of $V$. parahaemolyticus. No activity was detected in $V$. alginolyticus $10-1$ even at the initial cell number of $10^{5}$. All the strains used gave the highest activity when the initial cell number was $10^{7}$. The results suggest that the cell number of various $V$. parahaemolyticus can be estimated by measuring trypsin-like activity after cultivation in BSP-HP medium, though the selective-enrichment medium for V. parahaemolyticus needs to be improved.

Elimination of the interference by food-derived enzymes

It is assumed that enzymes in the food may interfere with the measurement of Vibrioderived enzyme. In fact, when enzyme activities in oysters were measured, high arylaminopeptidase and trypsin-like activities were observed in the absence of Vibrio species. To avoid

Table 4. Effects of EDTA on Arylaminopeptidase and Trypsin-Like Activity of Oyster and V. parahaemolyticus WP-1

\begin{tabular}{|c|c|c|c|c|}
\hline \multirow{3}{*}{ Sample } & \multicolumn{4}{|c|}{ Activity } \\
\hline & \multicolumn{2}{|c|}{ Arylaminopeptidase } & \multicolumn{2}{|c|}{ Trypsin-like } \\
\hline & None & $1 \mathrm{~m} M$ EDTA & None & $1 \mathrm{~m} M$ EDTA \\
\hline Control & 1.0 & 1.0 & 1.0 & 1.0 \\
\hline Oyster & 121.0 & 110.0 & 3.9 & 1.3 \\
\hline \multicolumn{5}{|l|}{ V. parahaemolyticus } \\
\hline $6.4 \times 10^{4}$ cells $/ \mathrm{ml}$ & 1.4 & 1.1 & 1.1 & 1.1 \\
\hline $6.4 \times 10^{5}$ cells $/ \mathrm{ml}$ & 2.2 & 1.2 & 2.5 & 2.9 \\
\hline $6.4 \times 10^{6}$ cells $/ \mathrm{ml}$ & 11.6 & 2.7 & 18.4 & 16.9 \\
\hline $6.4 \times 10^{7}$ cells $/ \mathrm{ml}$ & 122.0 & 27.8 & 90.0 & 120.0 \\
\hline
\end{tabular}

Table 5. Detection of $V$. parahaemolyticus in Seafoods by Fluorogenic Assay and BTB Teepol Agar Assay

\begin{tabular}{lccc}
\hline \hline \multirow{2}{*}{ Sample } & $\begin{array}{c}\text { Fluorogenic assay } \\
\text { (trypsin-like activity) }\end{array}$ & \multicolumn{2}{c}{ BTB teepol agar assay (cells/g) } \\
\cline { 3 - 4 } & & V. parahaemolyticus & V. alginolyticus \\
\hline Control & 1.0 & 0 & 0 \\
Adductor of she!lfish & 7.4 & 600 & 3,400 \\
Ark shell & 48.2 & 7,700 & 5,400 \\
& 1.0 & 100 & $<100$ \\
Boiled octopus & 1.4 & 100 & $<100$ \\
& 50.4 & 12,000 & $<100$ \\
Dried young sardine & 1.2 & $<100$ & $<100$ \\
& 3.2 & 600 & $<100$ \\
Horse mackerel & 101.5 & 27,000 & 280 \\
Sea bream & 1.9 & $<100$ & 7,200 \\
& 2.2 & 600 & 3,800 \\
Spratelloides gracilis & 25.9 & 8,900 & 300 \\
& 1.7 & 400 & 300 \\
Squid & 121.0 & 25,000 & $<100$ \\
& 0.8 & 100 & $<100$ \\
& 1.5 & 400 & $<100$ \\
& 41.8 & 14,000 & $<100$ \\
& 2.3 & 100 & $<100$ \\
& 4.5 & 700 & 9,000 \\
& 92.0 & 29,000 & 9,100 \\
\end{tabular}




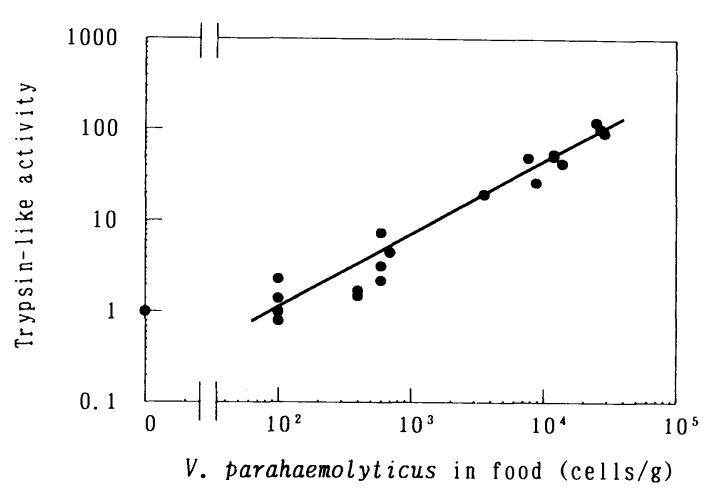

Fig. 3. Relationship between the cell number of $V$. parahaemolyticus in food and trypsin-like activity

V. parahaemolyticus was determined by the conventional BTB teepol agar plating method.

this problem, a specific inhibitor that inhibits the food enzymes but not Vibrio enzymes was required. Among the inhibitors tested, EDTA strongly inhibited the oyster trypsin-like enzyme but not the Vibrio trypsin-like enzyme (Table 4). EDTA did not inhibit the oyster arylaminopeptidase (Table 4). Thus, when $1 \mathrm{mM}$ EDTA was added to the reaction mixture, there was no interference in the activity of Vibrio trypsin-like enzyme by the food trypsin-like enzyme, and therefore, the trypsin-like activity could be used as a specific indicator to detect some Vibrio species including $V$. parahaemolyticus.

\section{Comparison between the fluorogenic assay and conventional BTB teepol agar plating}

The fluorogenic assay developed in this study was used to detect $V$. parahaemolyticus from commercial fish and shellfish. The results were then compared with those obtained by the conventional BTB teepol agar plating method. Samples of commercial horse mackerel, boiled octopus, squid, blue sprat, sea bream, ark shell, dried young sardine and adductor of shellfish were inoculated with 0 to $10^{4}$ cells of $V$. parahaemolyticus per gram. Sample solution $(1 \mathrm{ml})$ was added to BSP-HP medium $(6 \mathrm{ml})$ and cultured at $30^{\circ} \mathrm{C}$ for $6 \mathrm{hr}$. Trypsin-like activity was measured by the fluorogenic assay. The results obtained by both methods are summarized in Table 5 and Fig. 3. A very strong correlation was recognized (correlation coefficient $r=0.97$ ) between the colony count of $V$. parahaemolyticus and the trypsin-like activity measured by the present method. When the activity was over 2.0 (double that of the control), the sample was estimated to contain over 400 cells of $V$. parahaemolyticus per gram.

\section{Discussion}

Feng et $a l .{ }^{6)}$ and Moberg ${ }^{7)}$ used glucuronidase as a marker enzyme for the detection of $E$. coli. The commercially available Biorapid E kit (Ajinomoto Co.) for rapid detection of $E$. coli measures the galactosidase activity of the bacteria after a selective enrichment culture. From the results with various microorganisms causing food poisoning and spoilage, very high glucuronidase and galactosidase activities were detected in E. coli (Table 2). The Biorapid B kit (Ajinomoto Co.) for total viable count contains several 7AMC derivatives of amino acids for detecting arylaminopeptidase activities of bacteria. In the present experiment, all bacteria tested exhibited arylaminopeptidase activity (Table 2). Our results support the utility of these enzyme tests for the detection of the bacteria described above. Phosphatase activity was detected in many gram-negative bacteria, $B$. cereus, and S. aureus (Table 2). By measuring the phosphatase activity, $S$. aureus is probably detected specifically when it is cultured in the enrichment medium that contains inhibitors of growth of gram-negative bacteria and $B$. cereus.

TCBS agar medium or BTB teepol agar medium has been used to identify $V$. parahaemolyticus. Horie et al.$^{8)}$ developed one specific medium by using the arabinose-degrading ability of this species, and Baross et al. ${ }^{9)}$ and Twedt $e t$ $a l{ }^{10)}$ developed another medium by using the starch-degrading ability. These reports have been focused on selective isolation media, upon which bacterial samples were spread directly on agar plates. These methods require at least 24 hr to obtain results. On the other hand, the fluorogenic assay developed in the present study requires less time-about $8 \mathrm{hr}$, including the enrichment culture period, and $V$. parahaemolyticus populations over 400 cells/g were detectable (Fig. 3).

In the present method of Vibrio detection, 
both the growth and trypsin-like activity of bacteria other than a few Vibrio species were suppressed in BSP-HP medium (Table 3). Since in this medium, $V$. alginolyticus and $V$. harveyi, as well as $V$. parahaemolyticus, grew vigorously and had high trypsin-like activity (Table 3 ), it was impossible to detect only $V$. parahaemolyticus specifically. However, V. parahaemolyticus infection is considered to be very frequent in foods with high trypsin-like activity. Therefore, the present method is useful as a rapid detection method for some Vibrio species including $V$. parahaemolyticus, for use in food quality control. The culture conditions and the specific medium that suppress $V$. alginolyticus and $V$. harveyi but enrich $V$. parahaemolyticus will be reported shortly.

\section{Acknowledgments}

The authors are deeply grateful to Prof. Y. Kuwabara, Kagawa Nutrition College, Prof. K. Shinagawa, Iwate University, Dr. H. Konuma, National Institute of Hygienic Science, Dr. M. Tokumaru, Saitama Institute of Public Health, Dr. S. Konno, Sendai Municipal Institute of Public Health and Dr. S. Umesako, Nara Prefec- tural Institute of Public Health for their invaluable suggestions during the present investigation.

\section{References}

1) Nakashima, S., Takimoto, K.: Food Sanitation Research 37(7), 50 76 (1987).

2) Pharmaceutical Society of Japan ed.: "Standard Methods of Analysis for Hygienic Chemistswith commentary -" p. 102 129 (1980), Kanehara Publishing Co., Tokyo.

3) Morichi, T.: Food Sanitation Research 34(7), 23 38 (1984).

4) Maddocks, J. L., Greenan, M. J.: J. Clin. Pathol. 28, 686 687 (1975).

5) Tsutsumi, M., Nishimura, K., Yasui, K., Matsuoka, A., Watanabe, T.: J. Food Hyg. Soc. Japan 17, 273 275 (1976).

6) Feng. P.C. S., Hartmann, P. A.: Appl. Environ. Microbiol. 43, 1,320 1,329 (1982).

7) Moberg, L. J.: ibid. 50, 1,383 1,387 (1985).

8) Horie, S., Yamada, Y., Tanaka, H., Yamashita, Y., Aihara, T.: J. Food Hyg. Soc. Japan 19, 383 391 (1978).

9) Baross, J., Liston, J.: Appl. Microbiol. 20, 179 186 (1970).

10) Twedt, R. M., Novelli, R. M. E.: ibid. 22, 593 599 (1971). 